University of Nebraska - Lincoln

DigitalCommons@University of Nebraska - Lincoln

Biological Systems Engineering: Papers and

Publications

Biological Systems Engineering

2011

\title{
In Vivo Magnetic Resonance Elastography of Mesenchymally Derived Constructs
}

\author{
Shadi F. Othman \\ University of Nebraska-Lincoln, sothman2@unl.edu \\ Evan T. Curtis \\ University of Nebraska - Lincoln, ecurtis@huskers.unl.edu \\ Huihui Xu \\ University of Nebraska - Lincoln, hxu7@unl.edu
}

Follow this and additional works at: https://digitalcommons.unl.edu/biosysengfacpub

Part of the Biological Engineering Commons

Othman, Shadi F.; Curtis, Evan T.; and Xu, Huihui, "In Vivo Magnetic Resonance Elastography of Mesenchymally Derived Constructs" (2011). Biological Systems Engineering: Papers and Publications. 233.

https://digitalcommons.unl.edu/biosysengfacpub/233

This Article is brought to you for free and open access by the Biological Systems Engineering at DigitalCommons@University of Nebraska - Lincoln. It has been accepted for inclusion in Biological Systems Engineering: Papers and Publications by an authorized administrator of DigitalCommons@University of Nebraska Lincoln. 


\title{
In Vivo Magnetic Resonance Elastography of Mesenchymally Derived Constructs
}

\author{
Shadi F. Othman, Evan T. Curtis, Huihui Xu* \\ Biological Systems Engineering \\ University of Nebraska - Lincoln \\ Lincoln, NE, USA \\ hxu7@unl.edu
}

\begin{abstract}
The multidisciplinary field of tissue engineering (TE) aims to create biological substitutes to restore, maintain, or enhance the function of tissue or whole organs, in which the mechanical properties of replacements are critical to their structure and function. Several techniques have been investigated for measuring the mechanical properties of developing tissues and organs; however, current methods are destructive. The goal of this work is to apply high resolution Magnetic Resonance Elastography ( $\mu \mathrm{MRE}$ ) technique with 100 $\mu \mathrm{m}$ in-plane resolution to address a critical problem in TE: nondestructive monitoring and characterization of growing mesenchymal stem cells (MSCs)-based tissue engineered constructs. In this study, $\mu$ MRE is used to assess the development of osteogenic and adipogenic constructs implanted in immunodeficient nude mice. In vivo MRE experiments were conducted at $9.4 \mathrm{~T}$ (400 $\mathrm{MHz}$ for protons) using an $89 \mathrm{~mm}$ vertical bore magnet. Multiple shear waves were presented in the adipogenic constructs in comparison to the osteogenic constructs. This study demonstrated that $\mu \mathrm{MRE}$ can be used to noninvasively monitor the growth and development of various types of engineered tissues in vivo. (Abstract)
\end{abstract}

Keywords - Tissue Engineering (TE); Magnetic Resonance Elastography (MRE); mechanical property; stiffness (key words)

\section{INTRODUCTION}

Approximately one in five people will require organ replacement therapy before reaching 65 years of age [1]. Physicians treat organ failure commonly either by surgically transplanting tissues or by replacing living tissues with mechanical/electrical devices. Relevant to this project are patients suffering burn injuries, cancer resections, bone and cartilage loss due to trauma or degenerative diseases. The need to diagnose and treat these conditions is crucial. Unfortunately, it is not always possible to achieve these goals with current technology and natural healing alone. In addition, the financial cost is unbearable. For example, approximately one million bone grafts are performed each year in the United States with estimated annual cost of more than \$3 billion [2]. Osteoarthritis, a leading chronic disability in the middle age population, affects approximately 70 million people [3 \& 4] and has an estimated annual cost of more than $\$ 65$ billion [5] in the United States alone. Finally, soft tissue defects present traumatic challenges. Breast and facial cancer leave patients with disfiguration after resection. Soft tissue reconstruction represents a widespread clinical need. Tissue engineering (TE) and regenerative medicine are emerging biomedical technologies that can help restore normal tissue function. A key aspect of TE is to ensure that the replacement tissues exhibit the structure and function of the original, of which the mechanical property is essentially crucial. For instance, the replacement tissues of skin burn or breast lump both require lipid, yet each has a distinct mechanical property due to structural constraint. Assessing the stiffness in a dynamic and non-invasive manner will increase the quality of engineered tissues, which increases the repeatability of targeted engineered tissue characters and reduces the possibility of poorly developed samples.

Magnetic Resonance Imaging (MRI) provides sensitive measures that include a number of inherent parameters, such as relaxation times, diffusion, etc., to evaluate the structural and compositional characteristics of degenerative or regenerative biological tissues [6]. By inducing shear wave motions in the biological samples non-destructively, Magnetic Resonance Elastography (MRE) introduces a new quantitative parameter which uniquely illustrates spatiallylocalized information on the mechanical properties of tissues [ 7 \& 8]. MRE has been investigated in various studies, such as the detection of breast cancer [9], the visualization of the elastic properties of the brain [10], and assessment of human hyaline cartilage [11]. Recently, MRE was extended to the microscopic scale where high resolution MRE ( $\mu \mathrm{MRE}$ ) was used to monitor small biological tissues [12].

Tissue engineering is a dynamic and comprehensive process and requires continuous assessment of the outcome for the engineered tissues. Generally, tissue engineers evaluate cell morphology and activity through the use of microscopy and histologic stains. While such biochemical analysis provides a representation of molecular content and signaling pathways - for example, staining for alkaline phosphatase (ALP) is an early osteogenic marker - it is

978-1- 61284-704-7/11/\$26.00 @2011IEEE 
destructive to the tissue [13]. Therefore, non-invasive imaging modalities can play a vital role in the assessment of engineered constructs. Figure 1 shows the principle of tissue engineering and how proposed MRE testing is combined as an evaluation tool for three types of engineered tissues originating from mesenchymal stem cells (MSCs) adipogenic, osteogenic, and chondrogenic tissues. In this paper, our focus is the in vivo monitoring of TE constructs implanted in an animal model.

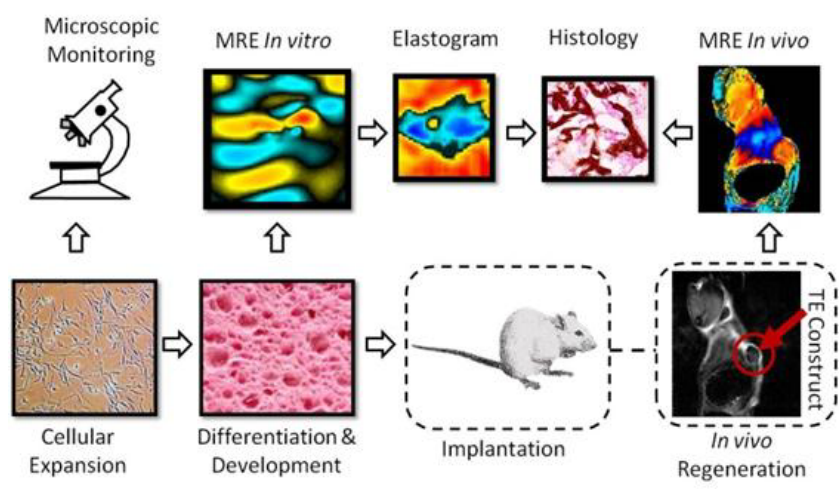

Figure 1. Flow diagram of the principle of tissue engineering. The proposed MRE techniques are used to monitor and assess the engineered constructs both in vitro and in vivo.

\section{Material ANd Methods}

\section{A. Construct Preparation}

First, tissue engineered constructs were prepared using mouse mesenchymal stem cells (mMSCs) (D1 ORL UVA, ATCC, Manassas, VA, USA). These constructs will be identified by mMSC at the end of the image caption. Healthy human bone $h \mathrm{MSCs}$ were isolated from fresh marrow cells provided commercially (Lonza, Walkersville, MD, USA). These constructs will be identified by hMSC at the end of the image caption. Upon $80 \%$ confluence, the MSCs were trypsinized, counted, and passaged at a density $5 \times 10^{3}$ cells $/ \mathrm{cm}^{2}$ and expanded in vitro. The medium was exchanged every other day. Expanded to passage 4-5 MSCs were trypsinized, counted, and re-suspended and seeded into gelatin sponges (Pharmacia \& Upjohn, Kalamazoo, MI, USA) with a dimension of $4 \times 4 \times 4 \mathrm{~mm}^{3}$ under vacuum induced by a $20 \mathrm{ml}$ syringe, followed by incubation in $5 \%$ $\mathrm{CO}_{2}$ at $37^{\circ} \mathrm{C}$ for $2 \mathrm{hrs}$. The TE constructs were kept in basic medium overnight to allow for complete cell adherence to the sponges. Beginning the next day as Day 0, the constructs were cultured in osteogenic and adipogenic medium. Osteogenic medium is a basic medium supplemented with $100 \mathrm{nM}$ dexamethasone, $10 \mathrm{mM} \beta$-glycerophosphate, and $0.05 \mathrm{mM}$ ascorbic acid-2-phosphate. Adipogenic medium is a basic medium supplemented with $50 \mathrm{nM}$ of dexamethasone, $10 \mu \mathrm{M}$ of insulin, and $5 \mathrm{mM}$ of isobutylmethylxanthine.

\section{B. Tissue Implantation}

All animal work was approved by the Animal Care Committee at UNL (University of Nebraska - Lincoln, Lincoln, NE, USA). Engineered tissue constructs are implanted subcutaneously in eight week old male nude immunodeficient mice (nu/nuJ, Jackson Laboratories, Bar Harbor, ME, USA). For the implantation surgery, the mouse was anesthetized with ketamine and xylazine at a dose of 90 $\mathrm{mg} / \mathrm{kg}$ and $10 \mathrm{mg} / \mathrm{kg}$, respectively. The surgical site was disinfected with betadine and isopropyl alcohol. A $20 \mathrm{~mm}$ incision was made mid-sagittal across the dorsum in the prescapular region where a subcutaneous pocket was created on the right side of the midline using blunt dissection. The construct was implanted and fixed in location by placing a suture through the muscle. Following the surgery, the animals were allowed to heal for two weeks before removing the suture and conducting MRE testing.

\section{MRI System and MRE Measumrents}

All MR experiments were conducted at $9.4 \mathrm{~T}(400 \mathrm{MHz}$ for protons) using an $89 \mathrm{~mm}$ vertical bore magnet (Agilent, Santa Clara, CA, USA). $\mu$ MRE measurements were acquired using a $4 \mathrm{~cm}$ Millipede RF imaging probe with triple axis gradients (maximum strength of $100 \mathrm{G} / \mathrm{cm}$ ). A phase contrast based pulse sequence was integrated with the imaging software, VnmrJ 2.3A, to enable the user to select MRE parameters, including the gradient amplitude (0-100 $\mathrm{G} / \mathrm{cm}$ ), the actuator frequency, the delay between the piezoelectric actuator and the bipolar gradient $(\psi)$, the bipolar pulses (MSG) direction, and the number of bipolar pairs.

A crucial step in MRE experiments is the characterization of the mechanical actuator for maximizing the motion induction in the tissues. The vibrations of the mechanical system were characterized using a Laser Doppler Vibrometer (Polytec, Dexter, MI, USA). The vibrometer measures the displacement of the actuator by pointing a Laser beam on its surface and analyzing the reflected signal. The frequency response is characterized by sending a white noise (signal composed by all frequencies in the $10 \mathrm{~Hz}-2 \mathrm{kHz}$ range) to the actuator and recording the measured displacement. The resonance frequencies of the system can be identified by observing the spectrum of the measured displacement, in which the output displacements at resonance frequencies are magnified. This procedure optimizes the motion of the actuator when generating waves with significant amplitudes in the engineered constructs. After being characterized, a signal generator is used to send signals at specific resonance frequencies; the output displacement is measured accordingly. The choice of the operating frequency is based on the displacement it provides, with the constraints of being within suitable range of MRE. 


\section{RESUlts}

Figure 2 shows a fast spin-echo MRI of a subcutaneous osteogenic construct four weeks post implantation in a mouse. The significant decrease of image intensity on the construct compared to neighboring soft tissues suggests bone formation. Following MRI, the construct was excised from the host mouse and assessed with hematoxylin and eosin staining confirming the bone differentiation.
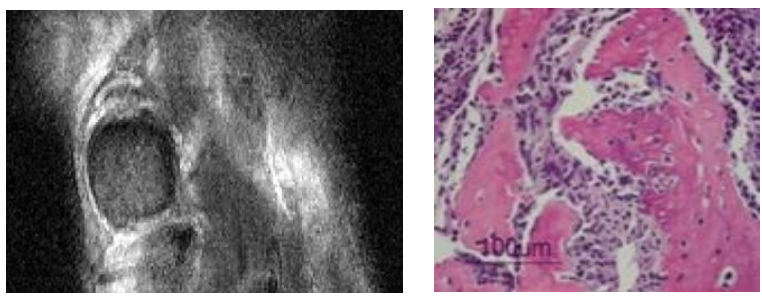

Figure 2. Osteogenic construct after four weeks of incubation. (Left) Fast spin-echo MRI. The construct, as circled, is approximately $3 \mathrm{~mm}$ in diameter. Imaging parameters are: matrix size $=2562$; slice thickness $=1.0$ $\mathrm{mm}$; repetition time $(\mathrm{TR})=2 \mathrm{~s}$; echo trail length $(\mathrm{ETL})=4$; echo spacing = $10 \mathrm{~ms}$; field-of-view $(\mathrm{FOV})=2.5 \mathrm{~cm} 2$; number of averages $=4$. The significant decrease of image intensity on the construct compared to neighboring tissues suggests bone formation, confirmed by (Right) hematoxylin \& eosin staining of excised tissue.

MR magnitude images of osteogenic and adipogenic tissues were acquired at different in vivo growth periods as shown in Figure 3, where the constructs are clearly delineated and significant difference in signal density is observed between the two tissue types, suggesting different mechanical properties, measured by palpation as well.
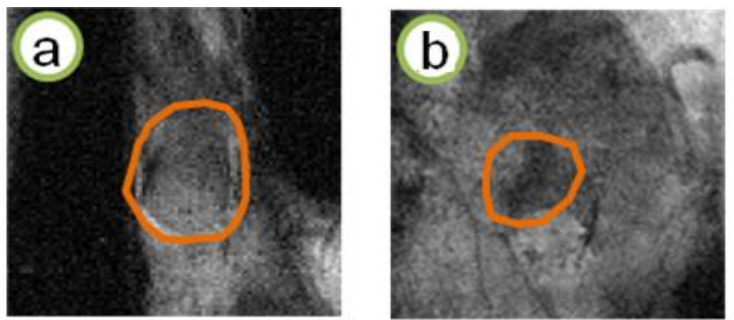

Figure 3. Adipose (a) and osteogenic (b) constructs (hMSC) two weeks after in vivo implantation. The circled areas indicate the engineered tissue constructs. Differences in signal intensity on the MR magnitude images are observed, due to different tissue type and various mechanical properties of the tissues.

Figure 4 shows the MR magnitude images and the corresponding shear wave images obtained through MRE measurements in osteogenic constructs two and four weeks post implantation. Notice that a full shear wave was not attained in the construct four weeks post implantation due to increased stiffness.

Interestingly, MRE studies on engineered adipogenic tissue construct demonstrated different result. As shown in Figure 5, multiple shear waves are observed in reconstructed MRE stiffness map through the adipogenic construct three weeks post implantation, corresponding to relatively lower tissue stiffness in comparison to osteogenic constructs.
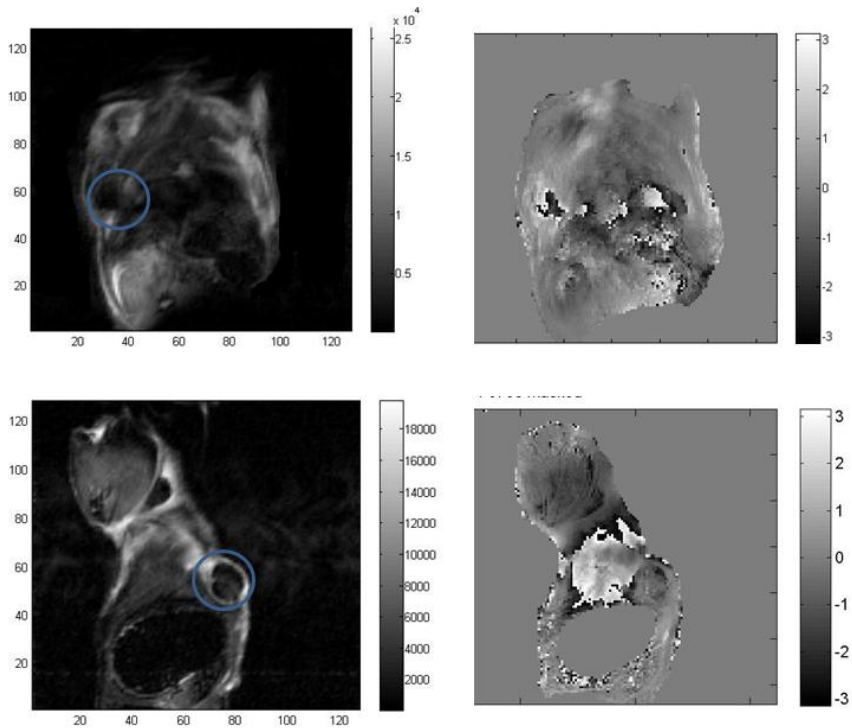

Figure 4. MR magnitude images (Left Column) and corresponding shear wave images (Right Column) of osteogenic constructs two (Top) and four (Bottom) weeks post implantation.

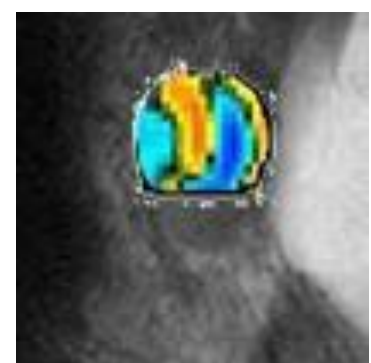

Figure 5. Reconstructed shear wave image through an adipogenic construct three weeks post implantation. Multiple shear waves can be recognized through the construct, suggesting lower tissue stiffness.

\section{DiscuSSION}

In MRE, strains associated with the wave propagation are measured using a phase contrast imaging technique, and these data are used to construct a stiffness map for a tissue's mechanical properties. This work addresses a critical problem in TE that involves the non-invasive assessment of resulting engineered tissue constructs by dynamically monitoring the mechanical properties in vivo. While no single imaging modality can fully represent the progressive process of tissue engineering, high resolution MRE technique is a potentially revolutionary development and can be used as a powerful complement to conventional biochemical analysis.

Increasing the dynamic range of measured stiffness by increasing the shear wave excitation frequency will allow the interrogation of stiffer materials. To further improve the 
faithfulness of the stiffness calculation, increasing the temporal frequency $f$ of the mechanical actuator should be considered. This will lead to a decrease of the wavelength $\lambda$, as the shear wave speed $c=f \lambda$ is directly related to the mechanical properties. Figure 6 describes the same experiment, using a two layer gel phantom consisting of two different concentrations of agarose gel, performed at two frequencies -400 and $800 \mathrm{~Hz}$. It can be noticed that the stiffness map calculated from the $800 \mathrm{~Hz}$ experiment is more accurate than the one obtained at the $400 \mathrm{~Hz}$, confirming the influence of the wavelength on the quality of the estimate of the mechanical stiffness. However, increasing the frequency presents one major drawback: waves will attenuate much faster, especially in viscous biological tissues. This requires the use of more powerful actuators and high voltage amplifier to provide motion with sufficient amplitude in the tissue of interest, and simultaneous excitations at multiple locations can also be considered to provide uniform wave patterns at frequencies higher than $1 \mathrm{kHz}$.

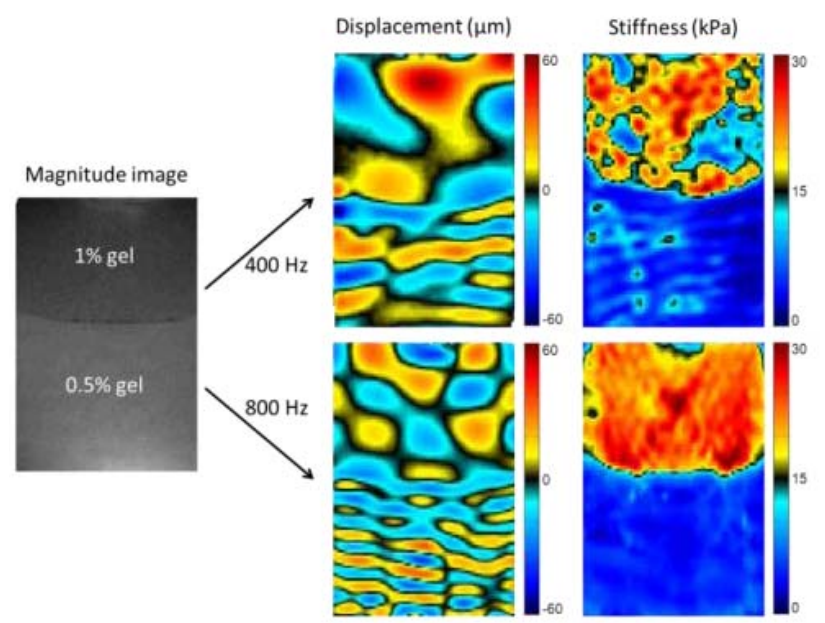

Figure 6. MRE experiment conducted at $400 \mathrm{~Hz}$ and $800 \mathrm{~Hz}$ on a gel phantom made of $1 \%$ and $0.5 \%$ wt agarose gel. Given the same sample being tested, results of $800 \mathrm{~Hz}$ are more accurate than $400 \mathrm{~Hz}$.

\section{ACKNOWLEDGMENT}

This research was supported in part by NIH -EB007299-02.

\section{REFERENCES}

[1] Lysaght, MJ and O'Loughlin, JA. 200. Demographic scope and economic magnitude of contemporary organ replacement therapies. ASAIO J. 46, 515-521

[2] Laurencin CT, Ambrosio AMA, Borden MD, and Cooper JA Jr. 1999. Tissue engineering: orthopedic applications. Annu. Rev. Biomed. Eng. 1:19-46

[3] Kraus VB. 1997. Pathogenesis and treatment of osteoarthritis. Med. Clin. North Am. 81:85-112

[4] O'Driscoll SW. 1998. The healing and regeneration of articular cartilage. J. Bone Joint Surg. Am. 80:1795

[5] Mao JJ. 2005. Stem-cell-driven regeneration of synovial joints. Biol. Cell 97:289-301

[6] Johnson GA, Hedlund LW, Cofer GP, and Suddarth SA. 1992. Magnetic Resonance Microscopy in the Life Sciences. Reviews of Magnetic Resonance in Medicine 4: 187-219

[7] Muthupillai R, Lomas DJ, Rossman PJ, Greenleaf JF, Manduca A, and Ehman RL. 1995. Magnetic resonance elastography by direct visualization of propagating acoustic strain waves. Science 269:18541857

[8] Muthupillai R, Rossman PJ, Lomas DJ, Greenleaf JF, Riederer SJ, and Ehman RL. 1996. Magnetic resonance imaging of transverse acoustic strain waves. Magn. Reson. Med. 36:266-274

[9] McKnight AL, Kugel JL, Rossman PJ, Manduca A, Hartmann LC, Ehman RL. 2002. MR elastography of breast cancer: preliminary results. AJR Am J Roentgenol 178(6):1411-7

[10] Braun J, Bernarding J, Tolxdorff T, Sack I. 2002. In Vivo Magnetic Resonance Elastography of the Human Brain Using Ultrafast Acquisition Techniques. Proc 10th Scient Meet ISMRM, Honolulu, 2597

[11] Lopez O, Amrami KK, Manduca A, Ehman RL.2008. Characterization of the dynamic shear properties of hyaline cartilage using high-frequency dynamic MR elastography. Magn Reson Med. 59(2):356-64

[12] Othman SF, Xu H, Royston TJ, and Magin RL. 2005. Microscopic magnetic resonance elastography (microMRE). Magnetic Resonance in Medicine 54:605-614

[13] Sabokbar A, Millett PJ, and Rushton N. 1994. A rapid, quantitative assay for measuring alkaline phosphatase activity in osteoblastic cells in vitro. Bone and Mineral. 27:57-67. 